\title{
Blocking IncRNA-SNHG16 Sensitizes Gastric Cancer Cells to 5-Fu Through Targeting the miR- 506-3p-PTBP1-Mediated Glucose Metabolism
}

\section{Yan Ding}

Tulane University of Louisiana: Tulane University

\section{Sujie Gao}

China-Japan Union Hospital of Jilin University

Jiabin Zheng

China-Japan Union Hospital of Jilin University

Xuebo Chen ( $\nabla$ chenxb@jlu.edu.cn )

China-Japan Union Hospital of Jilin University https://orcid.org/0000-0002-0069-8555

\section{Research}

Keywords: IncRNA SNHG16, PTBP1, Warburg effect, Glycolysis, 5-Fu resistance

Posted Date: August 6th, 2021

DOl: https://doi.org/10.21203/rs.3.rs-775664/v1

License: (9) This work is licensed under a Creative Commons Attribution 4.0 International License. Read Full License 


\section{Abstract}

Gastric cancer (GC) is one of the most human malignancies. The 5 -fluorouracil (5-Fu) is a first-line antigastric cancer chemotherapeutic agent. However, a large number of GC patients developed 5-Fu resistance. Currently, the biological roles and molecular mechanisms of IncRNA-SNHG16 in 5-Fu resistant gastric cancer still remain elusive. Here we report SNHG16 as well as PTBP1 are positively associated with 5-Fu resistance of gastric cancer. SNHG16 and PTBP1 are significantly upregulated in gastric tumors and cell lines. Silencing SNHG16 or PTBP1 effectively sensitized GC cells to 5-Fu. Furthermore, the glucose metabolism was remarkedly elevated in 5-Fu resistant GC cells. Under low glucose supply, 5-Fu resistant cells displayed higher vulnerability than parental GC cells. Bioinformatical analysis and luciferase assay demonstrated that SNHG16 downregulated miR-506-3p by sponging it. We identified PTBP1 was a direct target of miR-506-3p in GC cells. RNA-seq results indicated PTBP1 positively regulated multiple glycolysis enzymes expressions, including GLUT1, HK2 and LDHA. Bioinformatics analysis illustrated the $3^{\prime} U T R$ s of glycolysis enzymes contained multiple PTBP1 binding sites, which were further verified by RNA-pull down and RNA immunoprecipitation assays. Consequently, we demonstrated PTBP1 upregulated the mRNAs of glycolysis enzymes via promoting the mRNAs stabilities. Finally, in vivo xenograft experiments validated that blocking the SNHG16-mediated miR-506-3p-PTBP1 axis effectively sensitized xenograft tumors to 5-Fu treatments. Summarily, our study reports biological roles and molecular mechanisms underlying the IncRNA SNHG16-mediated 5-Fu resistance in GC through modulating the miR-506-3p-PTBP1-glycolysis axis, presenting a promising prospect in improvement of 5Fu therapy for chemoresistant GC patients.

\section{Introduction}

Gastric cancer (GC), one of the most common malignancies, is the second leading cause of mortality due to its tumorigenesis, development and metastasis $(1,2)$. Although the clinically surgical and chemotherapeutic approaches have been improved, most GC patients are diagnosed in advanced and/or metastatic stages, leading to disappointing prognosis (3). 5-Fluorouracil (5-Fu) is the first-line GC chemotherapeutic agent functioning through blocking DNA synthesis of tumor cells via inhibiting the thymidylate synthase activity (4). However, development of drug resistance has arisen as a major obstacle for the 5-Fu-based chemotherapy (5). Thus, it is urgent to investigate the underlying molecular mechanisms and develop effectively anti-chemoresistant approaches against 5-Fu resistance.

Long non-coding RNAs (IncRNAs), a novel class of noncoding RNAs, are endogenous RNAs with relatively large size (exceeding 200 nucleotides) (6). Accumulating studies revealed that IncRNAs play vital roles in diverse tumor progressions including tumorigenesis, migration, metabolism, and chemoresistance (7). LncRNA SNHG16 (small nucleolar RNA host gene 16) is a member of the small nucleolar RNA host gene family which is highly expressed in multiple cancers (8). For instance, studies reported that SNHG16 is high-expressed in colon cancer and regulated by the Wnt pathway (9). In addition, silencing SNHG16 inhibits prostate cell growth (10). Studies have uncovered a IncRNA-miRNA network where IncRNAs function as competitive endogenous RNAs (ceRNA) of miRNAs to recover the mRNA target genes 
expressions (11), suggesting the IncRNA-miRNA network is a potential target for anti-cancer therapy. Currently, the precise biological roles and molecular targets of SNHG16 in 5-Fu resistant gastric cancer have not been elucidated.

Tumor cells display a propensity that they metabolize glucose anaerobically rather than aerobically accompanying increased lactate production, even under enough oxygen supply (12). This phenomenon is known as "Warburg Effect" (13). Moreover, this feather of cancer cells is tightly correlated to chemoresistance (14). We and other colleagues have reported that 5-Fu resistant cancer cells displayed upregulated glycolysis and could be re-sensitized by blocking glycolysis (15), suggesting reversing the Warburg effect could effectively enhance the therapeutic outcomes of traditional anti-cancer agents. Polypyrimidine tract binding protein (PTBP1) is an RNA-binding protein acting as a splicing factor involving in diverse biological processes (15). Particularly, PTBP1 has been reported to function as an important regulator in cancers, including glioma (16), breast cancer (17), gastric cancer (18), lung cancer (19), renal cell carcinoma (20) and colon cancer (21). Studies have proven that PTBP1 has the capacity to promote glycolysis through modulating the pyruvate kinase M2 isoform (PKM2), which is a critical regulator of glycolysis $(22,23)$. However, the precise molecular mechanisms for the PTBP1-regulated glycolysis and the roles of PTBP1 in 5-Fu resistance in gastric cancer cells has not yet been elucidated.

In this study, the biological roles of IncRNA SNHG16 and PTBP1 in 5-Fu resistant gastric cancer will be investigated. The miRNA targets of SNHG16 will be identified. Furthermore, we assessed the mechanisms for the PTBP1-mediated glucose metabolism in gastric cancer. The combined anti-tumor effects by PTBP1 inhibition plus 5-Fu treatments will be verified using in vitro and in vivo models. Our study described a non-coding RNA-based therapeutic strategy as well as its underlying molecular mechanism, providing a promising therapeutic approach to overcome 5-Fu resistance in gastric cancer.

\section{Results}

\section{LncRNA-SNHG16 is significantly upregulated in gastric cancer and positively correlated with PTBP1 expression}

Accumulating evidence revealed that IncRNA SNHG16 and PTBP1 are apparently associated with poor prognosis and malignant phenotype of multiple cancers $(8-10,15)$, we started to investigate the clinical relevance of SNHG16 and PTBP1 in gastric cancer. Totally Fifty-five gastric tumor tissues and their corresponding adjacent normal tissues were collected and the expressions of SNHG16 and PTBP1 were examined by qRT-PCR. We observed that the SNHG16 expressions were significantly upregulated in gastric tumor tissues compared with normal tissues (Fig. 1A, S1A). Consistently, Kaplan-Meier Plotter survival analysis from the kmplot.com indicated high SNHG16 expressions were significantly associated with pool survival rates of gastric cancer patients (Fig. 1B). Meanwhile, with the comparison of SNHG16 in normal gastric epithelial cells, GES-1 and other five gastric cancer cell lines, we found SNHG16 was remarkedly upregulated in gastric cancer cells (Fig. 1C). These results suggest SNHG16 plays an oncogenic role in gastric cancer. Similarly, PTBP1 mRNA and protein expressions were obviously 
increased in gastric tumors compared with normal gastric tissues by qRT-PCR and IHC (Fig. 1D, 1E, S1B). Moreover, high PTBP1 expressions were associated with pool survival rates of gastric cancer patients (Fig. 1F). Consistent results from Fig. 1G showed that PTBP1 was significantly upregulated in gastric cancer cells. Intriguingly, bioinformatics analysis from StarBase revealed an apparently positive correlation between SNHG16 and PTBP1 in gastric tumors (Fig. 1H). To assess the regulatory relationship between them, gastric cancer cells AGS and SGC-7901 were transfected with SNHG16 siRNA or control siRNA. As we expected, silencing SNHG16 effectively attenuated PTBP1 expressions (Fig. 1I). Taken together, the above results demonstrated IncRNA SNHG16 upregulated PTBP1 in gastric cancer cells and positively associated with gastric tumor progress.

\section{SNHG16 and PTBP1 promote 5-Fu resistance of gastric cancer cells}

The acquired chemoresistance which frequently occurs in gastric cancers has attracted global attention. To explore the roles of SNHG16 and PTBP1 in 5-Fu resistance, we assessed the effects of silencing SNHG16 and PTBP1 on 5-Fu sensitivity of gastric cancer cell. SNHG16 was effectively knocked down in AGS and SGC-7901 cells by siRNA (Fig. 2A). AGS and SGC-7901 cells with normal or low SNHG16 expression were treated with elevated concentrations of 5-Fu for 48 hours. As we expected, gastric cancer cells with either SNHG16 or PTBP1 silencing displayed increased 5-Fu sensitivity compared with control cells (Fig. 2B, 2C, 2D). The 5-Fu IC50s of AGS was $138.75 \mu \mathrm{M}$. Silencing SNHG16 or PTBP1 effectively dropped IC50s of them to 47.42 or $41.72 \mu \mathrm{M}$ (Fig. 2B, 2C, 2D). Consistent results were obtained from SGC-7901 cells that knocking down SNHG16 or PTBP1 significantly sensitized GC cells to 5- Fu (Fig. 2E, $2 \mathrm{~F}, 2 \mathrm{G})$.

To gain an in-depth understanding the roles of SNHG16 and PTBP1 in 5-Fu resistant gastric cancer, we established a 5-Fu resistant gastric cancer cell line from AGS cell by gradually exposing cells to increasing concentrations of 5-Fu for three months. To verify the 5-Fu resistance, AGS parental and resistant cells were treated with 5-Fu at 0, 40, 80, 160, 320 or $640 \mu \mathrm{M}$. As shown in Fig. 2G, parental AGS cells, which have a 5-Fu IC50 at $134.22 \mu \mathrm{M}$, showed reduced cell viabilities with 5-Fu treatments. The 5-Fu resistant AGS cells could tolerate higher concentrations of 5-Fu treatments (IC50: $463.12 \mu \mathrm{M}$ ) (Fig. 2G). Expectedly, SNHG16 and PTBP1 were significantly upregulated in 5-Fu resistant cells (Fig. 2H, 2I). In summary, these results demonstrated SNHG16 and PTBP1 are positively associated with 5-Fu resistance in gastric cancer, suggesting targeting SNHG16 and PTBP1 could contribute to enhance the cytotoxicity of chemotherapeutic agents.

\section{5-Fu resistant GC cells exhibits upregulated glycolysis rate}

Mounting studies revealed the cancer cell displayed dysregulated glucose metabolism which affected chemosensitivity of multiple anti-cancer drugs (14). To investigate the mechanisms behind the SNHG16mediated 5-Fu resistance, we evaluated the glycolytic phenotypes of parental and 5-Fu resistant gastric cancer cells through Seahorse Extracellular Flux analysis. Dynamically bioenergetic results demonstrated 
that 5-Fu resistant cells displayed significantly increased extracellular acidification rate (ECAR) and an overall glucose uptake compared with AGS parental cells (Fig. 3A, 3B). Consistently, expressions of glycolysis key enzymes, GLUT1, Hexokinase 2 (HK2) and Lactate dehydrogenase-A (LDHA) were significantly upregulated in 5-Fu resistant gastric cancer cells (Fig. 3C). On the contrary, the mitochondrial respiration rate (OCR) was decreased in 5-Fu resistant cells (Fig. S2), indicating a reverse "Warburg effect" was observed in 5-Fu resistant gastric cancer cells. To assess the effects of the elevated glucose metabolism in 5-Fu resistant cells, AGS parental and 5-Fu resistant cells were cultured under regular and low glucose medium for 48 hours. Under low glucose, in contrast to parental cells which exhibited attenuated growth rate, 5-Fu resistant cells showed more severe growth inhibition (Fig. 3D). Expectedly, under 5-Fu treatment, 5-Fu Res cells displayed significantly increased cell death under low glucose compared with parental cells (Fig. 3E), indicating a glucose-addictive characteristic of 5-Fu resistant GC cells. To investigate whether targeting the increased cellular glycolysis rate by glycolysis inhibitors could sensitize 5-Fu resistant cells, we co-treated AGS 5-Fu resistant cells with 2-DG or Oxamate, two glycolysis inhibitors with 5-Fu. Results in Fig. 3F, 3G and 3H clearly demonstrated that blocking glycolysis remarkedly enhanced the 5-Fu sensitivity of AGS 5-Fu resistant cells. Taken together, these results illustrated targeting glycolysis could be an effective approach to overcome chemoresistance.

\section{SNHG16 positively associates with glycolysis of GC cells}

The above results revealed correlations between the SNHG16 and PTBP1-mediated 5-Fu resistance and upregulated cellular glycolysis of 5-Fu resistant gastric cancer cell. To test whether SNHG16 could regulate glycolysis, the AGS cells were transfected with control siRNA or SNHG16 siRNA. The glycolytic phenotype (ECAR) of SNHG16 silencing cells was significantly decreased through Seahorse Extracellular Flux analysis (Fig. 4A). In addition, silencing SNHG16 effectively blocked the glucose uptake (Fig. 4B) and glucose metabolism key enzymes, GLUT1, HK2 and LDHA expressions (Fig. 4C, 4D). Consistently, bioinformatics analysis demonstrated that SNHG16 expressions are positively associated with glycolysis enzymes expressions from the TCGA gastric cancer database (Fig. S3A-S3C). These results suggest targeting SNHG16 could effectively inhibit the upregulated glucose metabolism of 5-Fu resistant GC cells.

\section{miR-506-3p has tumor suppressive roles in gastric cancer and is sponged by SNHG16}

In order to evaluate the mechanisms for the SNHG16-promoted 5-Fu resistance of gastric cancer, we investigated the molecular targets of SNHG16. Studies revealed that IncRNAs function as ceRNA of miRNAs through direct sponging with them (11). Bioinformatical analysis predicted that SNHG16 could potentially bind seeding region of miR-506-3p (Fig. 5A). Moreover, literature research revealed miR-506-3p was negatively correlated with progresses of multiple cancer types (24-26), suggesting miR-506-3p might be a downstream molecule of SNHG16 and involve in 5-Fu resistance. To test this, gastric tumor tissues and their corresponding adjacent normal tissues were collected and the expressions of miR-506$3 p$ were examined by qRT-PCR. Expectedly, SNHG16 were significantly downregulated in gastric tumor 
tissues compared with normal tissues (Fig. 5B). Kaplan Meier plotter analysis indicated high miR-506-3p expression was associated with a better survival rate of gastric cancer patients (Fig. 5C). Moreover, a significantly invert expression pattern was observed between SNHG16 and miR-506-3p in gastric tumors (Fig. 5D). These results demonstrated miR-506-3p plays a tumor suppressive role in gastric cancer. To further assess the role of miR-506-3p in 5-Fu resistance, we compared the miR-506-3p expression between AGS parental and 5-Fu resistant cells. MiR-506-3p was found to be downregulated in 5-Fu resistant cells (Fig. 5E). In addition, gastric cancer cells with miR-506-3p overexpression showed increased sensitivity to 5-Fu (Fig. 5F, 5G), indicating specific delivering miR-506-3p to local tumors could effectively enhance chemotherapy. We then asked whether SNHG16 acts as a ceRNA of miR-506-3p in gastric cancer cells. Silencing of SNHG16 apparently increased miR-506-3p expressions in gastric cancer cells (Fig. 5H). To validate the direct binding of SNHG16/miR-506-3p, a luciferase assay was performed by constructing luciferase reporter vectors containing original (WT) or miR-506-3p binding site mutated (Mut) SNHG16 (Fig. 5A). Results clearly illustrated that co-transfection of miR-506-3p and WT SNHG16 could blocked the luciferase activity but not the binding site mutant SNHG16, (Fig. 5I). Collectively, the above results demonstrated miR-506-3p could sensitize gastric cancer cell to 5-Fu and function as a ceRNA of SNHG16.

\section{miR-506-3p inhibits cellular glycolysis through direct targeting PTBP1}

Studies reported that microRNAs function by binding to the $3^{\prime} U T R$ of target mRNAs (6). Thus, we analyzed the potential targets of miR-506-3p in gastric cancer. Bioinformatic analysis via the TargetScan.com indicated 3'-UTR of human PTBP1 contains a putative miR-506-3p binding region (Fig. 6A). Overexpression of miR-506-3p effectively blocked PTBP1 protein expressions in gastric cancer cells by Western blot (Fig. 6B). To verify whether miR-506-3p could directly bind to the 3'-UTR region of PTBP1 mRNA, AGS and SGC-7901 cells were co-transfected with of control miRNAs, miR-506-3p and 3'UTR of WT-PTBP1 or binding site mutant (Mut) PTBP1. As we expected, luciferase assay showed exogenous miR-506-3p significantly inhibited the activity of WT-PTBP1 3'-UTR but not the Mut PTBP1 3'UTR (Fig. 6C, 6D). We further detected a significantly reverse correlation between miR-506-3p and PTBP1 mRNA expressions in gastric cancer tissues (Fig. 6E). These results consistently validated PTBP1 could be directly targeted by miR-506-3p in gastric cancer. We next evaluated whether the miR-506-3p-mediated 5-Fu sensitivity was through targeting PTBP1. Consequently, rescue experiments were performed by cotransfection of miR-506-3p plus PTBP1 overexpression plasmid into AGS and SGC-7901 cells (Fig. 6F). Western blot results demonstrated successful rescue of PTBP1 expressions (Fig. 6F). Importantly, restoration of PTBP1 in miR-506-3p overexpressing gastric cancer cells recovered the glucose uptake and lactate product (Fig. 6G, 6H). Consequently, AGS and SGC-7901 cells with recovery of PTBP1 successfully rescued 5-Fu resistant phenotypes (Fig. 6I, 6J). Taken together, the above date demonstrated the miR-5063p-promoted 5-Fu sensitization of gastric cancer cells was through direct targeting PTBP1. 


\section{PTBP1 promotes glycolysis through modification of glycolysis enzymes expressions}

We then asked whether PTBP1 modulates glucose metabolism rate of gastric cancer cells. Bioinformatics analysis from TCGA gastric cancer database illustrated that PTBP1 expressions are positively associated with glycolysis enzymes expressions (Fig. S4A-S4C). As we expected, AGS cells with PTBP1 silencing showed significantly attenuated ECAR (Fig. 7A), glucose uptake (Fig. 7B) and glycolysis enzymes expressions (Fig. 7C). To further understand the molecular mechanisms for the PTBP1-promoted glycolysis, we performed RNA-Seq experiments by comparison of GC cells with control or PTBP1 silencing (Fig. 7D, 7E) to explore the transcriptional regulation by PTBP1. Following results of RNA-Seq, we analyzed the genes which were differentially expressed in PTBP1-silenced GC cells compared with control GC cells. From the totally 21221 genes, we identified 1067 genes that were regulated by PTBP1 knockdown (Fig. 7F). Among them, glycolysis enzymes, GLUT1, HK2 and LDHA were consistently downregulated by PTBP1 silencing (Fig. 7F), suggesting PTBP1 plays critical roles in the transcriptional regulation of glycolysis key enzymes. This regulation was further validated by qRT-PCR from AGS cells (Fig. S5). To reveal the potential biological roles of these DEGs (Differentially expressed genes), we subjected all 1067 DEGs to GO (Fig. 7G) and KEGG (Fig. S6) annotation. On the base of the cutoff criterion, the upregulated and downregulated genes were respectively enriched in GO terms. In the biological process terms of analysis, the upregulated genes in the PTBP1-KD cells mainly enriched in extracellular matrix organization, transmembrane transport, cell adhesion, synaptic transmission, insulin secretion, and intracellular signal transduction (Fig. 7G, S6). The downregulated genes mostly related to regulation of apoptotic process, G-protein coupled receptor signal transduction, energy reserve metabolic process, calcium signaling pathway and synaptic transmission (Fig. 7G, S6). Notably, GO annotation illustrated that PTBP1 silencing negatively affected the metabolic process, consistent with the abovedescribed biological functions of PTBP1 in glucose metabolism of GC cells. Taken together, these results demonstrated PTBP1 promoted glucose metabolism rate of gastric cancer cells through upregulating the glycolysis key enzymes transcriptions.

\section{PTBP1 stabilizes mRNAs of glycolysis enzymes via direct binding to 3'UTR regions}

Previous studies uncovered that PTBP1 functions as an RNA-binding protein to post-transcriptionally regulate target genes expressions (15). Thus, we analyzed the differentially regulated glycolysis enzyme mRNAs by PTBP1. Bioinformatics analysis of the PTBP1 mRNA targets from starBase predicted bindings of PTBP1 on 3'UTRs of glycolysis enzymes, GLUT1, HK2 and LDHA (Fig. S7A-S7C). Furthermore, among the predicted multiple PTBP1 binding motifs, we observed the 3'UTRs of GLUT1, HK2 and LHDA contained conserved PTBP1 binding motifs (Fig. 8A). Since previous studies described that PTBP1 associates with $3^{\prime} U T R$ of target mRNAs to enhance mRNA stability (15), we thus hypothesized PTBP1 enhances target mRNAs stability to upregulate glycolysis expressions by directly binding on $3^{\prime} U T R s$ of glycolysis enzymes. To evaluate that, RNA immunoprecipitation (RIP) assay was performed in AGS cells. 
qRT-PCR and RT-PCR results verified that the GLUT1, HK2 and LDHA mRNAs were apparently enriched in PTBP1 precipitated RNA fragments (Fig. 8B, 8C). We then performed RNA pull-down assay using biotinlabeled 3'UTRs of glycolysis enzymes. Western blot results in Fig. 8D showed significantly enrichment of PTBP1 which was associated with 3'UTRs of glycolysis enzymes. To evaluate whether the PTBP1upregulated glycolysis enzymes expressions through binding on 3'UTRs, AGS cells without or with PTBP1 knockdown were analyzed by RNA immunoprecipitation. Expectedly, AGS cells with PTBP1 silencing showed less amount of mRNA fragments immunoprecipitated by PTBP1 (Fig. 8E). The above results demonstrated specific associations between PTBP1 and 3'UTRs of glycolysis enzymes. To investigate whether PTBP1 affects mRNA stability of glycolysis enzymes, RNA stability assays were carried out to compare the half-lives of GLUT1, HK2 and LDHA mRNAs in control and PTBP1-silencing GC cells. As we expected, the half-lives of glycolysis enzyme mRNAs were significantly cut down in PTBP1-silencing cells compared with control cells (Fig. 8F, 8G, 8H). In summary, these results clearly validated that PTBP1 upregulated glycolysis enzyme expressions through direct bind to $3^{\prime} U T R$ s of them, resulting in the stabilization of enzyme mRNAs.

\section{Targeting the SNHG16-miR-506-3p-PTBP1 axis overcomes 5-Fu resistance in vitro and in vivo}

Given that SNHG16 and miR-506-3p-PTBP1-glycolysis inversely modulate 5-Fu sensitivity of gastric cancer, we examined whether SNHG16 accelerated 5-Fu resistance through suppressing the miR-506-3pPTBP1-glycolysis axis. Thus, AGS 5-Fu resistant cells were transfected with control, SNHG16 or SNHG16 plus miR-506-3p. Overexpression of SNHG16 significantly suppressed miR-506-3p expressions, which was further overridden by miR-506-3p transfection (Fig. 9A). Moreover, cells with SNHG16 plus miR-506$3 p$ transfection displayed restoration of PTBP1 (Fig. 9B), suggesting the SNHG16-mediated PTBP1 upregulation was through inhibiting miR-506-3p. Consequently, the ECAR (Fig. 9C), glucose uptake (Fig. 9D), and glycolysis enzymes (Fig. 9E) were effectively rescued by miR-506-3p restoration. Consequently, we observed overexpression of SNHG16 significantly de-sensitized AGS 5-Fu resistant gastric cancer cells (Fig. 9F, 9G). Yet, such de-sensitization was overridden by further miR-506-3p restoration (Fig. 9F, 9G), suggesting the SNHG16-regulated 5-Fu resistance was through modulating the miR-506-3p-PTBP1-glycolysis axis.

To validate the above proposed in vitro biological axis, a subcutaneous xenograft model was established. AGS 5-Fu resistant cells were stably infected with control or PTBP1 shRNA, followed by subcutaneously injection cells into the right flank of 6-week-old BALB/c nude mice. After establishment of xenograft tumors, mice without or with PTBP1 knockdown were treated with 5-Fu or control saline via intraperitoneal injection twice a week. As shown in Fig. 10A, most of the saline treated and control shRNA infected mice who received 5-Fu treatment died within 2 months. Although the PTBP1-knockdown mice without 5-Fu treatment showed slightly increased survival rate, the combination of PTBP1 knockdown and 5-Fu treatment achieved a significantly prolonged survival rate (Fig. 10A). Accordingly, consistent results demonstrated that the mice xenograft tumors underwent PTBP1 silencing plus 5-Fu treatment 
grew significantly slower than control and 5-Fu alone treatment (Fig. 10B, 10C). Moreover, qRT-PCR results from the collected mice tumors showed the mRNA expressions of GLUT1, HK2 and LDHA were apparently suppressed in xenograft tumors with PTBP1 knockdown plus 5-Fu treatment (Fig. 10D, 10E, 10F). Collectively, these results revealed that mice beard xenograft tumors from 5-Fu resistant gastric cancer cells with PTBP1 knockdown were more sensitive to 5-Fu treatment through inhibition of glycolysis, suggesting the combination of PTBP1 silencing with 5-Fu treatments renders a synergistically anti-tumor effect against chemoresistant gastric tumors.

\section{Discussion}

Gastric cancer is one of the prevalent malignancies in the world due to its tumorigenesis, development and metastasis $(1,2)$. Although chemotherapeutic approaches have been improved, a large fraction of GC patients developed drug resistance, which limited the effective applications of the 5-Fu-based chemotherapy. This study aimed to investigate the underlying molecular mechanisms and develop effectively anti-chemoresistant strategies against 5-Fu resistance. LncRNAs are known to serve as vital regulators of specific cellular processes (6). Moreover, IncRNAs are tightly associated with therapeutic options and prognostic values of cancer patients (6). Here we observed SNHG16 was positively associated with gastric cancer, consistently with previous reports $(9,10)$. Results from this study indicate that SNHG16 is overexpressed in the tumor tissues of GC patients and high expression of SNHG16 is associated with poor prognosis. Furthermore, silencing SNHG16 effectively sensitized 5-Fu resistant GC cells. Although previous studies have reported that SNHG16 is a gastric cancer cell-favorite molecule (27-29), our results for the first time demonstrated the roles of SNHG16 in 5-Fu resistance of gastric cancer. Given that IncRNAs function as competitive endogenous RNAs (ceRNA) of miRNAs by sponging them to suppress miRNA expression, leading to recovery of the miRNA target genes expressions, to investigate the molecular mechanisms, we identified miR-506-3p, which has been reported to function as a tumor suppressive miRNA in multiple cancers (24-26), was a directly downstream target of SNHG16 in GC cells by Western blot assay and luciferase assay. SNHG16 downregulates miR-506-3p expression through sponging it, suggesting the SNHG16-miR-506-3p ceRNA network could be a promising target for treatment of gastric cancer.

Chemoresistant cancer cells exhibit apparently metabolic reprogramming, a phenomenon is known as "Warburg Effect" $(13,14)$. Our previous studies have reported that 5-Fu resistant cancer cells displayed upregulated glycolysis and could be re-sensitized by blocking glycolysis (30). In this study, we described a SNHG16-miR-506-3p-PTBP1-glycolysis-5-Fu resistant axis in gastric cancer cells. SNHG16 and PTBP1 were significantly upregulated in 5-Fu resistant GC cells. In addition, silencing SNHG16 or PTBP1 effectively re-sensitized 5-Fu resistant cells. Mechanism rescue experiments showed co-transfection of SNHG16 with miR-506-3p effectively overrode the SNHG16-promoted PTBP1, glycolysis and 5-Fu resistance, suggesting the SNHG16-promoted 5-Fu resistance was through targeting the miR-506-3pPTBP1-glycolysis axis. 
Mounting evidence revealed that PTBP1 functions as a vital regulator for post-transcriptional gene expression (15-19). PTBP1 regulates mRNA expressions through messenger RNA (mRNA) splicing, localization, stability and translation (15-19). Existing evidence has suggested that PTBP1 upregulated pyruvate kinase 2 (PKM2), a glycolysis key enzyme, leading to a metabolic shift from oxidative phosphorylation to glycolysis in cancer cells $(22,23)$. Moreover, recent studies demonstrated the miR134/PTBP1 signaling cascade was involved in aerobic glycolysis to enhance osteosarcoma chemoresistance (31), indicating PTBP1 plays important regulatory roles in glucose metabolism. However, the precise molecular targets and underlying mechanisms remain elusive. We highlighted conserved PTBP1 binding motifs in 3'UTRs of major glycolysis enzymes, GLUT1, HK2 and LDHA. The associations between PTBP1 and the 3'UTRs of glycolysis enzymes were validated by RNA pull-down assay and RNA immunoprecipitation assay. Recent study uncovered that PTBP1 stabilizes mRNA by preventing the NMD protein UPF1 from binding 3'UTRs, resulting in evading recognition by the nonsensemediated mRNA decay pathway (32). We therefore performed the RNA stability assays and results showed the half-lives of glycolysis enzyme mRNAs were significantly cut down in PTBP1-silencing cells compared with control cells. These results clearly validated that PTBP1 upregulated glycolysis enzyme expressions through direct bind to 3'UTRs of them, resulting in the stabilization of enzyme mRNAs. Furthermore, mice xenograft results strongly supported the above in vitro conclusions. Xenograft tumors underwent PTBP1 silencing plus 5-Fu treatment grew significantly slower than control and 5-Fu alone treatment. Given that PTBP1 functions to regulate mRNA expressions at multiple post-transcriptional levels, the mechanisms underlying the regulation of glycolysis enzymes by PTBP1 are extensive and complex. Thus, other potential mechanisms by which PTBP1 promotes glycolysis are possible. Further investigation is required to address the detailed molecular mechanisms.

In summary, we demonstrated a promising prospect in improvement of 5-Fu therapy for GC patients. SNHG16 downregulates miR-506-3p as a ceRNA to de-repress its miRNA target, PTBP1, which upregulates glycolysis key enzymes expressions via binding to the 3'UTRs of enzymes mRNAs, leading to 5-Fu resistance in gastric cancer cells. This study will contribute to the development of new therapeutic strategies against 5-Fu resistant gastric cancers.

\section{Materials And Methods}

\section{Patient samples}

A total of 55 gastric tumor tissues and matched adjacent normal tissues were evaluated in this study. Tissues were obtained from patients who underwent surgery at the Department of General Surgery, ChinaJapan Union Hospital of Jilin University, China between 2015 and 2018. After surgical dissection, patient samples were immediately frozen by liquid nitrogen and stored at $-80^{\circ} \mathrm{C}$. Patients did not receive chemoor radio- therapy before surgery. This study was approved by the ethics committee from the Institutional Review Board of the China-Japan Union Hospital of Jilin University, Changchun, Jilin Province, P. R. China. All participants gave written informed consent. 


\section{Cell culture and reagents}

Five gastric cancer cell lines (AGS, BGC-823, MKN-45, MGC-803 and SGC-7901) and one normal gastric mucosa endothelial cell line GES-1 were purchased from the Cell Research Institute of the Chinese Academy of Sciences (Shanghai, China). Cells were cultured in RPMI 1640 medium (Thermo Fisher Scientific, Inc., Carlsbad, CA, USA) with 10\% fetal bovine serum (FBS) (Thermo Fisher Scientific, Inc., Carlsbad, CA, USA) and 100 units $/ \mathrm{ml}$ penicillin plus $100 \mu \mathrm{g} / \mathrm{ml}$ streptomycin (Thermo Fisher Scientific, Inc., Carlsbad, CA, USA) at $37^{\circ} \mathrm{C}$ in a humidified cell culture incubator with $5 \% \mathrm{CO}_{2}$. The 5 -Fu resistant gastric cancer cell line AGS/5-Fu R was established according to previous description by continually exposing to increased concentrations of 5-Fu (30). The acquired 5-Fu resistant cells were re-selected by treating with 5-Fu at $300 \mathrm{uM}$ every two months. The rabbit anti-GLUT1 (\#73015), rabbit anti-Hexokinase 2 (\#2867), rabbit anti-LDHA (\#3582) and rabbit anti- $\beta$-actin (\#4970) antibodies were purchased from Cell Signaling Technology (Danvers, MA, USA). Mouse anti-PTBP1 (\#32-4800) monoclonal antibody was purchased from Thermo Fisher Scientific (Shanghai, China). 5-Fu, 2-DG and Oxamate were purchased from Sigma-Aldrich (Shanghai, China).

\section{Construction and transfections of Plasmid DNA, siRNA and miRNA}

The pCDNA3.1-PTBP1 overexpression plasmid was constructed according to previous description (33). Fragment encoding the full-length cDNA of PTBP1 was ligated into the pcNDA3.1 vector. The small interfere RNA (siRNA) targeting SNHG16 was designed and synthesized by Sangon Biotech (Shanghai, China). The PTBP1 shRNA was synthesized by ABLife Biotech (Wuhan, China). Silence sequence of PTBP1: GCGTGAAGATCCTGTTCAATA. Sense and antisense strands were annealed to be shRNA. Vector



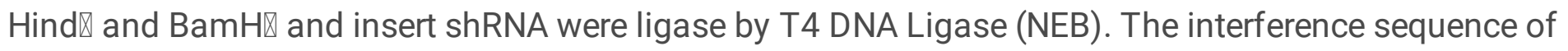
shRNA was verified by Sanger sequencing. Control miRNA and miR-506-3p precursor were purchased from GenePharma Co. (Shanghai, China). Transfections were performed using the Lipofectamine 2000 (Invitrogen, California, USA) according to the manufacturer's instructions. MiRNAs or siRNAs were transfected at $50 \mathrm{nM}$. Plasmid DNA was transfected at $2 \mu \mathrm{g}$. After $48 \mathrm{~h}$, cells were collected for the following experiments.

\section{RNA isolation and qRT-PCR}

Total RNA was isolated from surgically resected gastric tumors or cultured gastric cancer cells using the TRIZOL reagent (Ambion, USA). The RNA was further purified with two phenol-chloroform treatments and then treated with RQ1 DNase (Promega, Madison, WI, USA) to remove DNA. The quality and quantity of RNA samples were assessed by measuring the absorbance at $260 \mathrm{~nm} / 280 \mathrm{~nm}$ (A260/A280) using Smartspec Plus (BioRad, Hercules, CA, USA). First-strand cDNA was synthesized using 0.5 ug total RNA using a SuperScript First-Standard Synthesis System for RT-PCR (Invitrogen, Carlsbad, CA, USA) following the manufacturer's protocol. qRT-PCR experiments were performed using the SYBR Green qPCR Master Mix (ThermoFisher Scientific, Shanghai, China). Samples were analyzed using an ABI Prism 7700 
Sequence Detection System (Applied Biosystems, Foster City, NJ, USA). Primers for qRT-PCR were: miR506-3p: Forward: 5'- GCCACCACCATCAGCCATAC-3', Reverse: 5'-GCACATTACTCTACTCAGAAGGG-3', SNHG16: Forward: 5'-GCAGAATGCCATGGTTTCCC-3', Reverse: 5'- GGACAGCTGGCAAGAGACTT-3', PTBP1: Forward: 5'-GCATCGACTTTTCCAAGCTC-3', Reverse: 5'-GGAAACCAGCTCCTGCATAC-3', GLUT1 Forward: 5'- TTGCAGGCTTCTCCAACTGGAC-3', Reverse: 5'-CAGAACCAGGAGCACAGTGAAG-3', HK2: Forward: 5'TACACTCAATGACATCCGAACTG-3', Reverse: 5'-CGTCCTTATCGTCTTCAATATCC-3', LDHA: Forward: 5'ATGAAGGACTTGGCGGATGA-3', Reverse: 5'-ATCTCGCCCTTGAGTTTGTCTT-3', $\beta$-actin: Forward: 5'CTGAGAGGGAAATCGTGCGT-3', Reverse: 5'-CCACAGGATTCCATACCCAAGA-3'. The expression of $\beta$-actin was used to normalize the relative expression levels. Human U6 was an internal control for miRNA detection. The thermal profile was set as follows: $95^{\circ} \mathrm{C}$ for $1 \mathrm{~min}$ and 40 cycles at $95^{\circ} \mathrm{C}$ for $15 \mathrm{~s}, 58^{\circ} \mathrm{C}$ for $20 \mathrm{~s}$, and $72^{\circ} \mathrm{C}$ for $20 \mathrm{~s}$. Experiments were performed in triplicate and repeated three times. The results were analyzed using the $2^{-\Delta \Delta C t}$ method.

\section{RNA-Seq analysis}

The quality and quantity of the purified RNA samples were redetermined by measuring the absorbance at $260 \mathrm{~nm} / 280 \mathrm{~nm}$ (A260/A280) using Smartspec Plus (BioRad, USA). The integrity of RNA was further verified by $1.5 \%$ agarose gel electrophoresis. For each sample, $1 \mu \mathrm{g}$ of the total RNA was used for RNA-seq library preparation by VAHTS Stranded mRNA-seq Library Prep Kit (Vazyme Biotech Co., Ltd, Nanjing, China). Polyadenylated mRNAs were purified and fragmented, and then converted into double strand cDNA. After the step of end repair and A tailing, the DNAs were ligated to VAHTS RNA Adapters (Vazyme Biotech Co., Ltd, Nanjing, China). Purified ligation products corresponding to 200-500 bps were digested with heat-labile UDG, and the single strand cDNA was amplified, purified, quantified and stored at $-80^{\circ} \mathrm{C}$ before sequencing. For high-throughput sequencing, the libraries were prepared following the manufacturer's instructions and applied to Illumina HiSeq X Ten system for 150 nt paired-end sequencing.

\section{RNA-Seq raw data clean and alignment}

Raw reads containing more than 2-N bases were first discarded. Then adaptors and low-quality bases were trimmed from raw sequencing reads using FASTX-Toolkit (Version 0.0.13). The short reads less than 16nt were also dropped. After that, clean reads were aligned to the GRch38 genome by tophat2 (34) allowing 4 mismatches. Uniquely mapped reads were used for gene reads number counting and FPKM calculation (fragments per kilobase of transcript per million fragments mapped).

\section{Functional enrichment analysis}

To sort out functional categories of DEGs, Gene Ontology (GO) terms were identified using KOBAS 2.0 server (35). Hypergeometric test and Benjamin-Hochberg FDR controlling procedure were used to define the enrichment of each term.

\section{Differentially Expressed Genes (DEG) analysis}


The R/Bioconductor package edgeR (36) was utilized to screen out the differentially expressed genes (DEGs). A false discovery rate $<0.05$ and fold change $>2$ or $<0.5$ were set as the cut-off criteria for identifying DEGs.

\section{Bioinformatics analysis}

The interaction between IncRNA-SNHG16 and miR-506-3p was predicted by starBase of ENCORI (http://starbase.sysu.edu.cn/). The correlation between SNHG16 and PTBP1 expressions in gastric cancer patient was analyzed by starBase of ENCORI. The binding of miR-506-3p and PTBP1 was predicted from starBase of ENCORI. Survival rates according to SNHG16, miR-506-3p and PTBP1 expressions in gastric cancer patients were analyzed by www.kmplot.com. 3'UTRs of glycolysis enzymes were obtained from NCBI. Binding motifs of PTBP1 were analyzed from starBase of ENCORI. $p<0.05$ was considered statistical significance.

\section{RNA immunoprecipitation (RIP)}

RNA was isolated from GC cells using a Rneasy Mini kit (Qiagen, Hilden, Germany). GC cells were lysed in RIP lysis buffer from the Magna RIPTM RNA-binding protein immunoprecipitation kit (Millipore, Bedford, MA, USA). Anti-IgG (ab172730, Abcam) or anti-PTBP1 (\#32-4800, Thermofisher) antibody with A/G immunomagnetic beads were premixed in immunoprecipitation buffer. Cell lysates were mixed with above reaction to immuno-precipitate PTBP1-RNA complexes at $4{ }^{\circ} \mathrm{C}$ for 16 hours. Proteinase $\mathrm{K}$ was then added into the reaction. RNA samples were purified. The enrichments of GLUT1, HK2 and LDHA mRNAs which bond with PTBP1 were analyzed by qPCR and RT-PCR. Experiments were repeated three times.

\section{RNA pull-down assay}

RNA pull-down assay was performed according to previous report. Briefly. 3'UTRs of glycolysis enzymes and the negative control (antisense $3^{\prime} U T R$ and scramble) were in vitro transcribed and biotin labeled using a biotin RNA labeling mix (Roche, Shanghai, China). Mixture was treated with RNase-free DNase I and purified by a RNeasy mini kit (Qiagen, Hilden, Germany). Proteins from GC cell extracts were incubated with the above biotinylated RNAs at $50 \mathrm{pmol} / \mathrm{mg}$ followed by incubation with streptavidin agarose beads (Invitrogen, Carlsbad, CA, USA). After washing, protein samples were detected by Western blot. Experiments were repeated three times.

\section{RNA stability assay}

GC cells were first transfected with control siRNA or siPTBP1 for 48 hours. Actinomycin D (ActD) was added at a final concentration of $5 \mu \mathrm{g} / \mathrm{ml}$ and incubated for various periods. Total RNA was isolated and transcripts of glycolysis enzymes were examined by qRT-PCR. $\beta$-actin was an internal control.

\section{Seahorse metabolic flux analysis}


Extracellular acidification rate (ECAR) and oxygen consumption rate (OCR) were detected using the Seahorse XFp Analyzer (Agilent, Santa Clara, CA, USA) and detection kits from Agilent Technologies Inc. (Santa Clara, CA, USA) according to the manufacturer's instructions. Equal number of cells from each treatment were analyzed. Results were normalized by protein concentrations. Experiments were performed in triplicate and repeated three times.

\section{Glucose uptake and lactate production}

Gastric cancer cells were seeded on a 12-well plate $\left(1 \times 10^{5}\right.$ per well). The detections of glucose uptake and lactate production were performed using the Glucose Uptake Colorimetric Assay Kit (Applygen Technologies, Beijing, China) and the L-lactate assay kit (BioVision, Milpitas, CA, USA) according to the manufacturer's instructions. Results was normalized by the cell number of each well. Relative glycolysis rate was calculated from the absorbance of drug-treated cells/untreated cells. Experiments were performed in triplicate and repeated three times.

\section{Luciferase assay}

Gastric cancer cells $\left(5 \times 10^{4}\right.$ cells/well) were seeded in 24-well plates and cultured for 24 hours. Cells were then co-transfected with control miRNA or miR-506-3p plus $50 \mathrm{ng}$ pGL3-reporter luciferase reporter containing WT- or Mut- SNHG16 or PTBP1 3'UTR using Lipofectamine 2000 (Thermo Fisher Scientific Inc., Waltham, MA, USA). Forty-eight hours post-transfection, luciferase activity was measured using a Dual luciferase reporter assay system (Promega, Madison, WI, USA) according to the manufacturer's instructions. Firefly luciferase activity was normalized to that of the Renilla luciferase. Experiments were performed in triplicate and repeated three times.

\section{Cell viability assay}

Cell viability was examined by MTT (3-(4,5-dimethylthiazol-2-yl)-2,5-diphenyltetrazolium bromide) assay (Sigma-Aldrich, Shanghai, China). Gastric cancer cells $\left(5 \times 10^{3}\right.$ cells/well) were seeded into 96 -well plates at $80 \%$ confluence for 24 hours. Cells were washed with PBS. medium was refreshed and MTT solution was added at $37^{\circ} \mathrm{C}$ for 2 hours. Then, $150 \mu$ DMSO was added to dissolve the formazan crystals. The optical density (OD) of formazan concentrations was determined at $570 \mathrm{~nm}$ using a microplate reader (Bio-Rad Laboratories). The OD values were normalized by cell numbers. Relative viability was obtained from the absorbance at $540 \mathrm{~nm}$ of drug-treated cells/the absorbance at $540 \mathrm{~nm}$ of untreated cells. Experiments were performed in triplicate and repeated three times.

\section{Clonogenic assay}

Anchorage-dependent GC cells $\left(1 \times 10^{3}\right.$ cells/well) were plated onto 6-well plate for 24 hours. After 5-Fu treatment, medium was refreshed and cells were cultured for additional 10 days then stained by $1 \%$ crystal violet solution. The survival clones were examined under microscopy. Experiments were repeated three times. 


\section{Cell apoptosis assay}

GC Cells $\left(3 \times 10^{5}\right.$ per well) were seeded onto 6-well plates for 24 hours. Cell apoptosis rate was assessed by FITC-Annexin V/PI Kit (\#556547, BD Biosciences, San Jose, CA, USA) according to the manufacturer's instructions. Briefly, after treatments, cells were washed twice with cold PBS. Cells were stained with FITCAnnexin $\mathrm{V}(5 \mathrm{ml})$ and $\mathrm{Pl}(5 \mathrm{ml})$ for $30 \mathrm{~min}$ at room temperature in dark. Apoptosis was analyzed using a BD Accuri C6 Flow cytometer (BD Biosciences, San Jose, CA, USA). Experiments were repeated three times.

\section{Western blot}

Proteins were extracted from cells using the RIPA lysis buffer (Beyotime Ltd., Shanghai, China) suppled with $1 \mathrm{x}$ protease inhibitor cocktail (Sigma-Aldrich, Shanghai, China). Lysates were incubated on ice for 15 minutes and centrifugated at $10,000 \mathrm{~g}$ for $15 \mathrm{~min}$ at $4{ }^{\circ} \mathrm{C}$. Protein concentrations were measured by Bradford method. Equal amounts of proteins were applied to a $10 \%$ sodium dodecyl sulfatepolyacrylamide gel (SDS-PAGE) and transferred to a nitrocellulose membrane. Membranes were blocked with $5 \%$ nonfat milk at room temperature for 1 hour. After complete washing by PBST, membranes were incubated with primary antibodies (1:1000 dilution) with gentle shaking at $4{ }^{\circ} \mathrm{C}$ overnight. After washing, membranes were incubated with HRP-conjugated goat anti-mouse or goat anti-rabbit antibody (1:3000 dilution) at room temperature for 1 hour. The detection of antibody-bound protein signals was performed using the enhanced chemiluminescence kit (Bio-Rad Ltd., USA). Experiments were repeated three times.

\section{Xenograft mice model}

All of the xenograft experiments were complied with the guidelines of the Institutional Animal Care and Use Committee of the China-Japan Union Hospital of Jilin University. Totally thirty-two six-week-old nude mice were used in this study. Mice were kept on a regular 12/12 hr light-dark cycle cages. Nude mice were separated to two groups ( 16 each) then were injected subcutaneously with AGS cells $\left(8 \times 10^{6}\right)$ transfected with control shRNA or PTBP1 shRNA to establish xenograft models. Mice from each xenograft group were randomly divided into two groups and treated as follows: PBS control, $5-\mathrm{Fu}$ alone [ $40 \mathrm{mg} / \mathrm{kg}$ intraperitoneal (i.p.), 2 times/wk], sh PTBP1 alone and 5-Fu plus sh PTBP1. Mice mortality was monitored daily. Tumors from dead mice were collected at the death time point. After 80 days the survival mice were euthanized by $\mathrm{CO}_{2}$ method and the xenograft tumor tissues were dissected. Tumors were stored at $-80^{\circ} \mathrm{C}$ for downstream analysis. Experimental protocol was carried out in accordance with the European Communities Council Directive of 24 November 1986 (86/609/EEC) and approved by an institutional review committee from Institutional Animal Care and Use Committee of the China-Japan Union Hospital of Jilin University.

\section{Statistical analysis}

Statistical difference was analyzed using the GraphPad Prism 7.0 software. Results are presented as the mean \pm SD. The unpaired Student's t-test was used for the data analysis between two groups and 
significance among three or more groups was analyzed by one-way ANOVA followed by Bonferroni corrections. All experiments were performed in triplicate and repeated three times. A statistical difference of $p<0.05$ was considered significant.

\section{Declarations}

\section{Acknowledgements}

Authors express their gratitude to all the medical doctors, research scientists and staffs from the Department of General Surgery, China-Japan Union Hospital of Jilin University, Jilin, China for their supporting and advising during the conduct of this study.

\section{Author contributions}

Y.D. and X.B.CH. designed the present study, all authors performed the experiments and analyzed the data, Y.D. and X.B.CH. prepared and reviewed the manuscript. All authors read and approved the final manuscript.

\section{Conflict of research interests}

The authors declare that they have no competing interests.

\section{Funding}

This work was supported by the Natural Science Foundation of Science and Technology Development Plan in Jilin Province, China (1. No. 20180101303JC, 2. No. 202512JC010473382).

\section{Data availability statement}

The datasets used and/or analyzed during the current study are available from the corresponding author on reasonable request.

\section{References}

1. Smyth EC, Nilsson M, Grabsch HI, van Grieken NC, Lordick F. Gastric cancer. Lancet. 2020, 396: 635648.

2. Johnston FM, Beckman M. Updates on Management of Gastric Cancer. Curr Oncol Rep. 2019, 21: 67.

3. Biagioni A, Skalamera I, Peri S, Schiavone N, Cianchi F, Giommoni E, Magnelli L, Papucci L. Update on gastric cancer treatments and gene therapies. Cancer Metastasis Rev. 2019 Sep,38(3):537-548.

4. Longley DB, Harkin DP, Johnston PG. 5-fluorouracil: mechanisms of action and clinical strategies. Nat Rev Cancer. 2003, 3: 330-338.

5. Shi WJ, Gao JB. Molecular mechanisms of chemoresistance in gastric cancer. World J Gastrointest Oncol. 2016, 8: 673-681. 
6. Goodall GJ, Wickramasinghe VO. RNA in cancer. Nat Rev Cancer. 2021, 21: 22-36.

7. Peng WX, Koirala P, Mo YY. LncRNA-mediated regulation of cell signaling in cancer. Oncogene. 2017, 36: 5661-5667.

8. Gong CY, Tang R, Nan W, Zhou KS, Zhang HH. Role of SNHG16 in human cancer. Clin Chim Acta. 2020, 503: 175-180.

9. Wu W, Guo L, Liang Z, Liu Y, Yao Z. Lnc-SNHG16/miR-128 axis modulates malignant phenotype through WNT/beta-catenin pathway in cervical cancer cells. J Cancer. 2020, 11: 2201-2212.

10. Shao M, Yu Z, Zou J. LncRNA-SNHG16 Silencing Inhibits Prostate Carcinoma Cell Growth, Downregulate GLUT1 Expression and Reduce Glucose Uptake. Cancer Manag Res. 2020, 12: 17511757.

11. Paraskevopoulou MD, Hatzigeorgiou AG. Analyzing MiRNA-LncRNA Interactions. Methods Mol Biol. 2016, 1402: 271-286.

12. Abbaszadeh Z, Çeşmeli S, Biray Avcı Ç. Crucial players in glycolysis: Cancer progress. Gene. 2020, 726: 144158.

13. Koppenol WH, Bounds PL, Dang CV. Otto Warburg's contributions to current concepts of cancer metabolism. Nat Rev Cancer. 2011, 11: 325-37.

14. Ma L, Zong X. Metabolic Symbiosis in Chemoresistance: Refocusing the Role of Aerobic Glycolysis. Front Oncol. 2020, 10: 5.

15. Zhu W, Zhou BL, Rong LJ, Ye L, Xu HJ, Zhou Y, Yan XJ, Liu WD, Zhu B, Wang L, Jiang XJ, Ren CP. Roles of PTBP1 in alternative splicing, glycolysis, and oncogensis. J Zhejiang Univ Sci B. 2020, 21: 122-136.

16. Zhu L, Wei Q, Qi Y, Ruan X, Wu F, Li L, Zhou J, Liu W, Jiang T, Zhang J, Yin B, Yuan J, Qiang B, Han W, Peng X. PTB-AS, a Novel Natural Antisense Transcript, Promotes Glioma Progression by Improving PTBP1 mRNA Stability with SND1. Mol Ther. 2019, 27: 1621-1637.

17. Wang $X$, Li Y, Fan Y, Yu X, Mao X, Jin F. PTBP1 promotes the growth of breast cancer cells through the PTEN/Akt pathway and autophagy. J Cell Physiol. 2018, 233: 8930-8939.

18. Ren ZH, Shang GP, Wu K, Hu CY, Ji T. WGCNA Co-Expression Network Analysis Reveals ILF3-AS1 Functions as a CeRNA to Regulate PTBP1 Expression by Sponging miR-29a in Gastric Cancer. Front Genet. 2020, 11: 39.

19. Cho CY, Chung SY, Lin S, Huang JS, Chen YL, Jiang SS, Cheng LC, Kuo TH, Lay JD, Yang YY, Lai GM, Chuang SE. PTBP1-mediated regulation of AXL mRNA stability plays a role in lung tumorigenesis. Sci Rep. 2019, 9: 16922.

20. Takai T, Tsujino T, Yoshikawa Y, Inamoto T, Sugito N, Kuranaga Y, Heishima K, Soga T, Hayashi K, Miyata K, Kataoka K, Azuma H, Akao Y. Synthetic miR-143 Exhibited an Anti-Cancer Effect via the Downregulation of K-RAS Networks of Renal Cell Cancer Cells In Vitro and In Vivo. Mol Ther. 2019, 27: 1017-1027. 
21. Wang ZN, Liu D, Yin B, Ju WY, Qiu HZ, Xiao Y, Chen YJ, Peng XZ, Lu CM. High expression of PTBP1 promote invasion of colorectal cancer by alternative splicing of cortactin. Oncotarget. 2017, 8: 36185-36202.

22. Taniguchi $\mathrm{K}$, Uchiyama $\mathrm{K}$, Akao Y. PTBP1-targeting microRNAs regulate cancer-specific energy metabolism through the modulation of PKM1/M2 splicing. Cancer Sci. 2021, 112: 41-50.

23. Calabretta S, Bielli P, Passacantilli I, Pilozzi E, Fendrich V, Capurso G, Fave GD, Sette C. Modulation of PKM alternative splicing by PTBP1 promotes gemcitabine resistance in pancreatic cancer cells. Oncogene. 2016, 35: 2031-2039.

24. Chen L, Wang X, Ji C, Hu J, Fang L. MiR-506-3p suppresses papillary thyroid cancer cells tumorigenesis by targeting YAP1. Pathol Res Pract. 2020, 216: 153231.

25. Wang Y, Lei X, Gao C, Xue Y, Li X, Wang H, Feng Y. MiR-506-3p suppresses the proliferation of ovarian cancer cells by negatively regulating the expression of MTMR6. J Biosci. 2019, 44: 126.

26. Xia XY, Yu YJ, Ye F, Peng GY, Li YJ, Zhou XM. MicroRNA-506-3p inhibits proliferation and promotes apoptosis in ovarian cancer cell via targeting SIRT1/AKT/FOXO3a signaling pathway. Neoplasma. 2020, 67: 344-353.

27. Pang W, Zhai M, Wang Y, Li Z. Long noncoding RNA SNHG16 silencing inhibits the aggressiveness of gastric cancer via upregulation of microRNA-628-3p and consequent decrease of NRP1. Cancer Manag Res. 2019, 11: 7263-7277.

28. Zhou C, Zhao J, Liu J, Wei S, Xia Y, Xia W, Bi Y, Yan Z, Huang H. LncRNA SNHG16 promotes epithelialmesenchymal transition via down-regulation of DKK3 in gastric cancer. Cancer Biomark. 2019, 26 : 393-401.

29. Wang X, Kan J, Han J, Zhang W, Bai L, Wu H. LncRNA SNHG16 Functions as an Oncogene by Sponging MiR-135a and Promotes JAK2/STAT3 Signal Pathway in Gastric Cancer. J Cancer. 2019, 10: 1013-1022.

30. Gao S, Song D, Liu Y, Yan H, Chen X. Helicobacter pylori CagA Protein Attenuates 5-Fu Sensitivity of Gastric Cancer Cells Through Upregulating Cellular Glucose Metabolism. Onco Targets Ther. 2020, 13: 6339-6349.

31. Zhang Q, Wu J, Zhang X, Cao L, Wu Y, Miao X. Transcription factor ELK1 accelerates aerobic glycolysis to enhance osteosarcoma chemoresistance through miR-134/PTBP1 signaling cascade. Aging (Albany NY). 2021, 13: 6804-6819.

32. Ge Z, Quek BL, Beemon KL, Hogg JR. Polypyrimidine tract binding protein 1 protects mRNAs from recognition by the nonsense-mediated mRNA decay pathway. Elife. 2016, 5: e11155.

33. Wang D, Zhang H, Li M, Frid MG, Flockton AR, McKeon BA, Yeager ME, Fini MA, Morrell NW, Pullamsetti SS, Velegala S, Seeger W, McKinsey TA, Sucharov CC, Stenmark KR. MicroRNA-124 controls the proliferative, migratory, and inflammatory phenotype of pulmonary vascular fibroblasts. Circ Res. 2014, 114: 67-78.

34. Kim D, Pertea G, Trapnell C, Pimentel H, Kelley R, Salzberg SL. TopHat2: accurate alignment of transcriptomes in the presence of insertions, deletions and gene fusions. Genome Biol. 2013, 14: 
R36.

35. Xie C, Mao X, Huang J, Ding Y, Wu J, Dong S, et al. KOBAS 2.0: a web server for annotation and identification of enriched pathways and diseases. Nucleic Acids Res. 2011, 39: 316-322.

36. Robinson MD, Mccarthy DJ, Smyth GK. edgeR: a Bioconductor package for differential expression analysis of digital gene expression data. Bioinformatics. 2010, 26: 139.

\section{Figures}

A

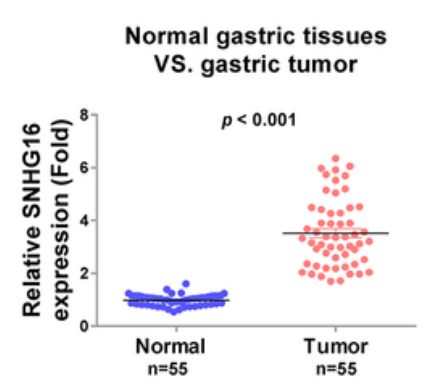

D

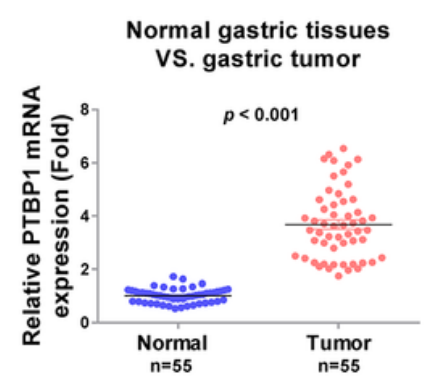

G

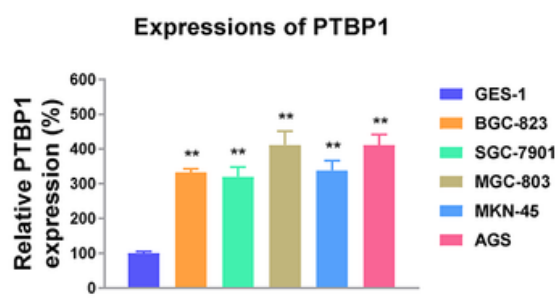

B

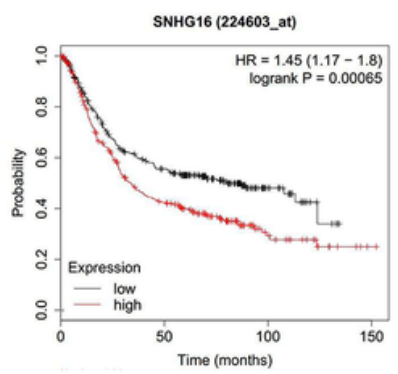

E

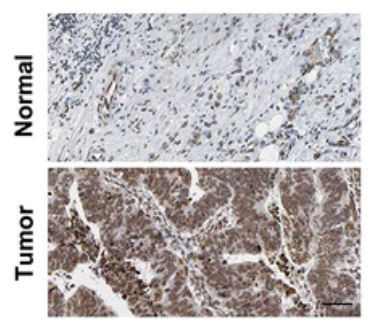

H

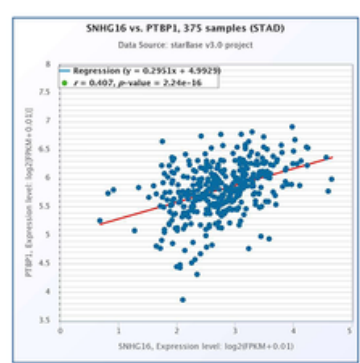

C

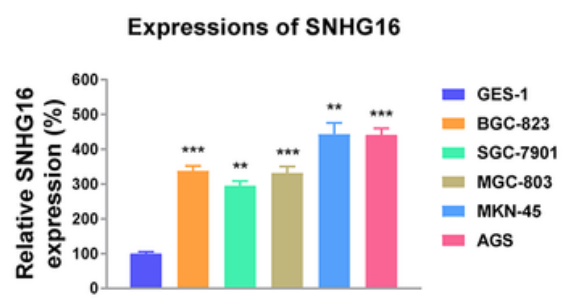

$\mathbf{F}$

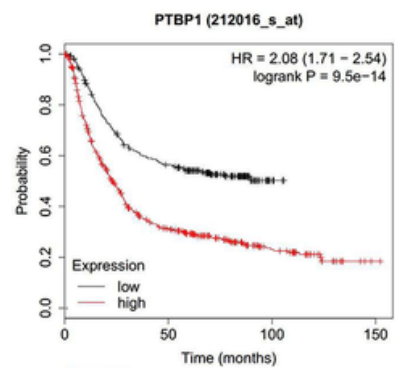

I

Figure 1

SNHG16 and PTBP1 are positively associated with gastric cancer. (A) SNHG16 expressions were detected in gastric tumors $(n=55)$ and their matched normal gastric tissues by qRT-PCR. (B) Kaplan-Meier Plotter analyzes the survival rates of GC patients with high or low SNHG16 expressions. (C) Expressions of SNHG16 were detected in one non-tumorigenic gastric epithelial cell line, GES-1 and five GC cell lines by qRT-PCR. (D) PTBP1 expressions were detected in gastric tumors $(n=55)$ and their matched normal gastric tissues by qRT-PCR and (E) IHC. (F) Kaplan-Meier Plotter analyzes the survival rates of GC patients with high or low PTBP1 expressions. (G) Expressions of PTBP1 were detected in one non-tumorigenic gastric epithelial cell line, GES-1 and five GC cell lines by qRT-PCR. (H) Pearson coefficient analysis shows a significantly positive correlation between SNHG16 and PTBP1 in gastric tumors. (I) AGS and SGC-7901 
cells were transfected with control or SNHG16 siRNA, protein expressions of PTBP1 were examined by Western blot. Data were presented as mean \pm S.D. *, $p<0.05, * *, p<0.01, * \star *, p<0.001$.

A

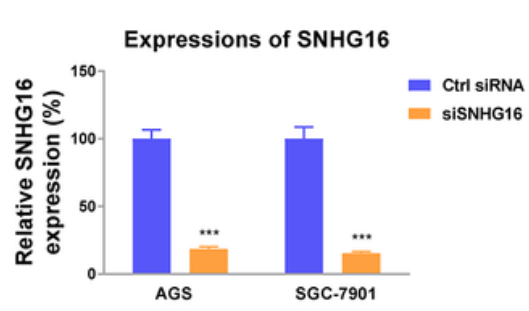

D

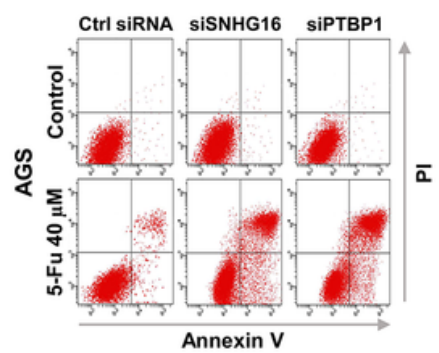

G

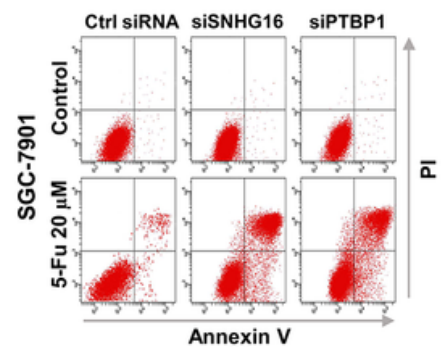

B

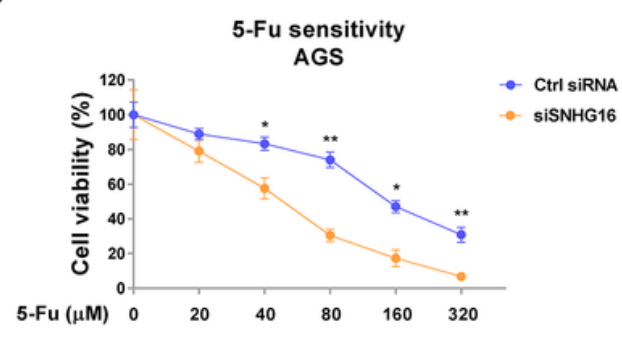

E

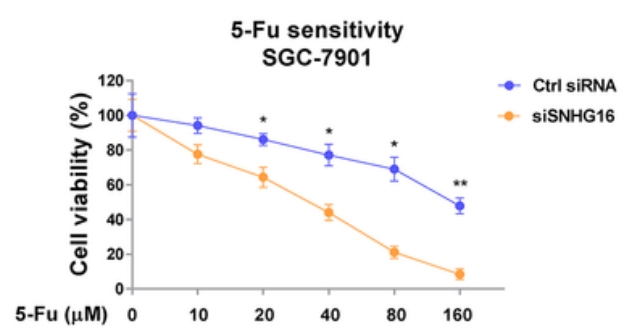

H



C

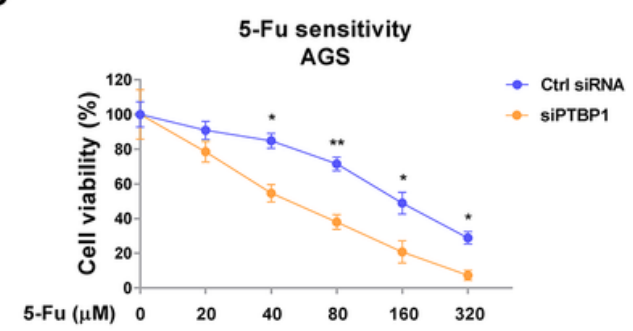

F

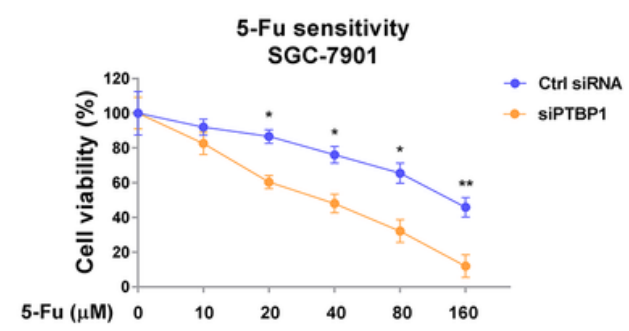

I

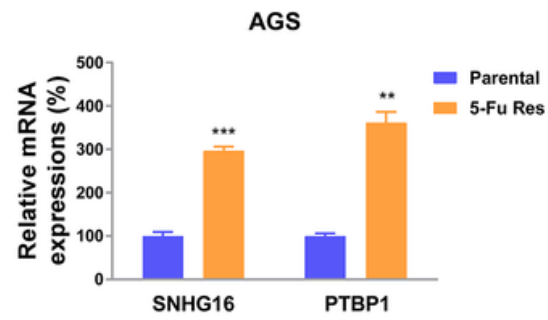

Figure 2

SNHG16 and PTBP1 promote 5-Fu resistance of gastric cancer. (A) AGS and SGC-7901 cells were transfected with control siRNA or SNHG16 siRNA, expressions of SNHG16 were examined by qRT-PCR. (B, C) AGS cells with SNHG16 or PTBP1 silencing were treated with 5-Fu at the indicated concentrations for 48 hours. Cell viability was determined by MTT assay and (D) Annexin V apoptosis assay. (E, F) SGC7901 cells with SNHG16 or PTBP1 silencing were treated with 5-Fu at the indicated concentrations for 48 hours. Cell viability was determined by MTT assay and (G) Annexin V apoptosis assay. (H) AGS parental cells and 5-Fu resistant cells were treated with 5-Fu for 48 hours. Cell viability was determined by MTT assay and clonogenic assay. (I) Expressions of SNHG16 and PTBP1 were examined in AGS parental and

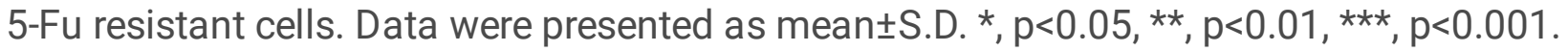


A

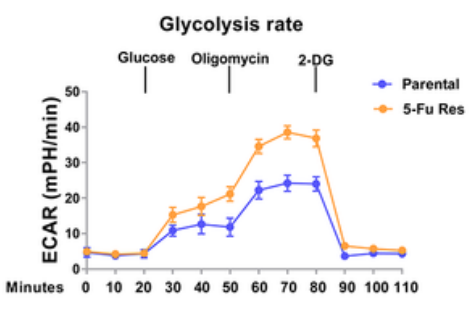

E

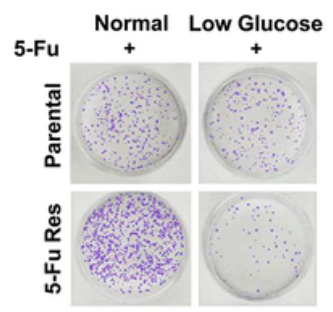

B

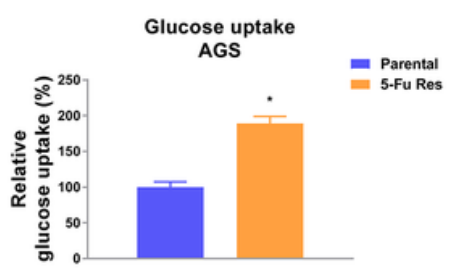

F

G

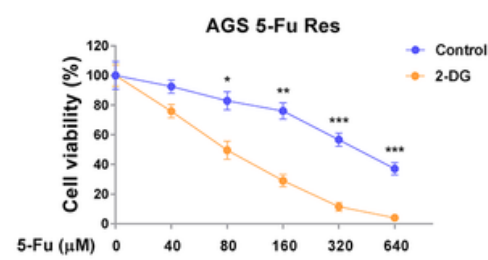

C

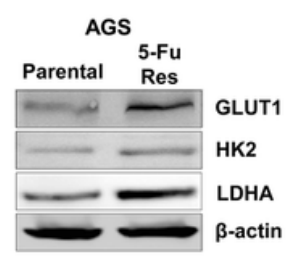

D

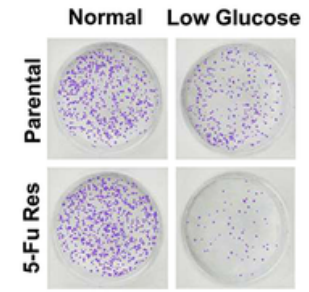

H

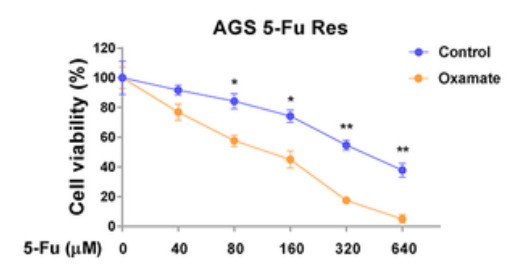

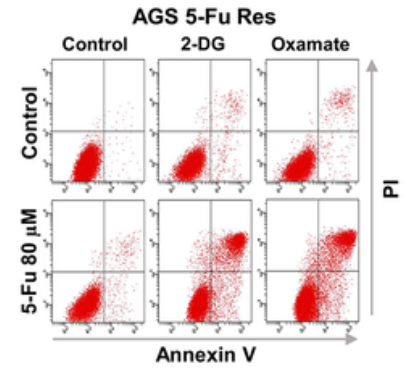

Figure 3

5-Fu resistant gastric cancer cells display glucose addictive phenotype. (A) ECAR, (B) glucose uptake and (C) glycolysis enzymes were measured in AGS parental and 5-Fu resistant cells. (D) AGS parental and 5Fu resistant cells were cultured under normal or low glucose conditions. Cell viability was determined by clonogenic assay. (E) The above cells under normal or low glucose conditions were treated with 5-Fu for 48 hours. Cell viability was determined by clonogenic assay. (F, G, H) AGS 5-Fu resistant cells were treated with 5-Fu alone or combined with glycolysis inhibitors at the indicated concentrations. Cell death was determined by MTT assay and Annexin $\mathrm{V}$ apoptosis assay. Data were presented as mean \pm S.D. ${ }^{*}, p<0.05$, $* *, p<0.01, * * *, p<0.001$.

A

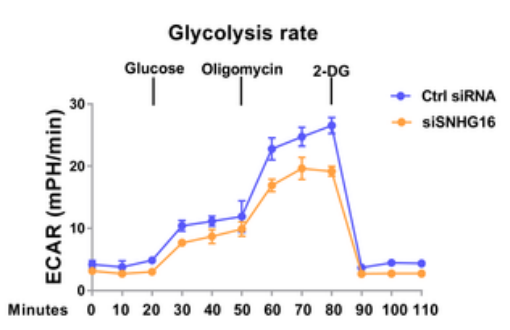

B

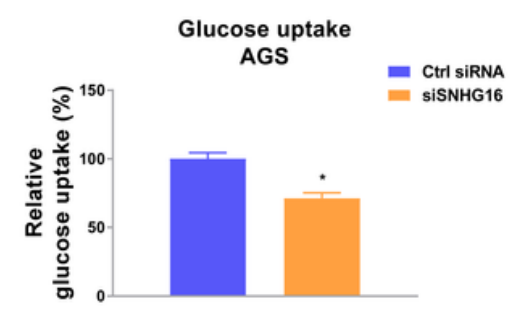

C

D



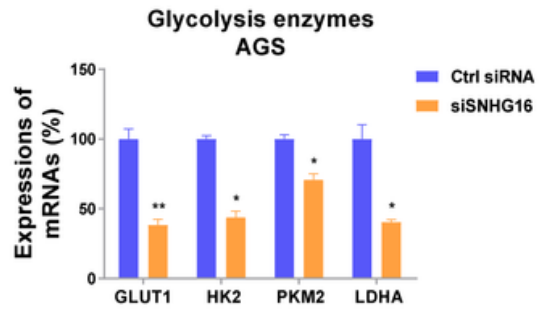

Figure 4

Effects of SNHG16 on glycolysis of GC cells. (A) AGS cells were transfected with control siRNA or SNHG16 siRNA. ECAR, (B) glucose uptake, (C, D) glycolysis enzymes were examined. Data were presented as mean \pm S.D. *, $p<0.05, * \star, p<0.01$. 
B

-506-3p-Inc SNGH16 Interactions Supported by Ago CLIP-seq Data

SNHG16: 5 ' guguCUCCGCAGAGUGCCUUC 3' ||| | || ||||||| miR-506-3p: $3^{\prime}$ agauGAGUCUUCCCACGGAAu 5'

D

SNHG16 vs. miR-506-3p gastric tumor

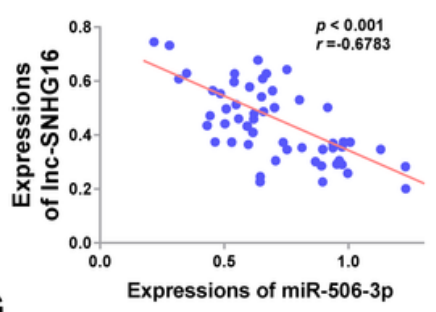

SGC-7901

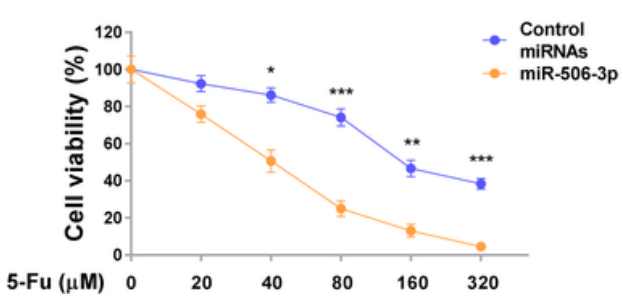

E


H



$\mathbf{F}$
C
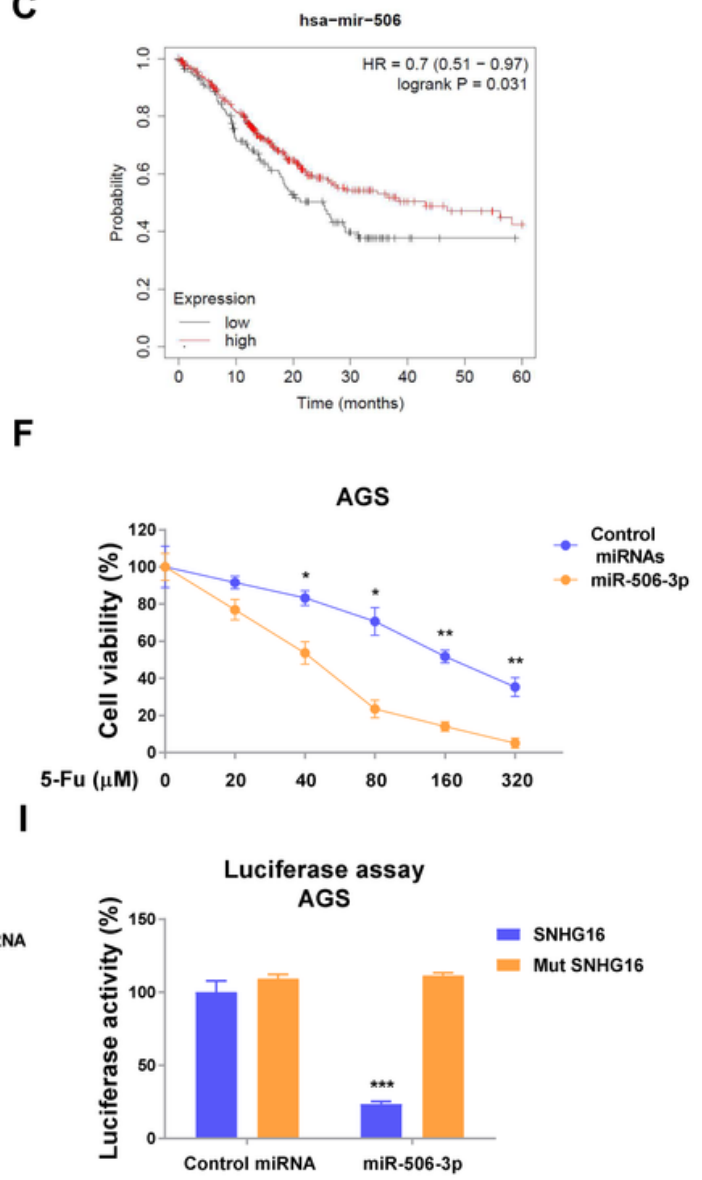

Figure 5

SNHG16 downregulates miR-506-3p in GC by sponging it. (A) The potential binding between SNHG16 and miR-506-3p was predicted from starBase. (B) miR-506-3p expressions were detected in gastric tumors $(n=55)$ and their matched normal gastric tissues by qRT-PCR. (C) Kaplan-Meier Plotter analyzes the survival rates of GC patients with high or low miR-506-3p expressions. (D) Pearson coefficient analysis shows a significantly negative correlation between SNHG16 and miR-506-3p in gastric tumors. (E) Expressions of miR-506-3p were detected in AGS parental and 5-Fu resistant cells. $(F, G)$ AGS and SGC7901 cells were transfected with control miRNA or miR-506-3p. Cells were treated with 5-Fu, cell viability were determined by MTT assay. $(H)$ GC cells were transfected with control or SNHG16 siRNA. Expressions of miR-506-3p were detected by qRT-PCR. (I) Luciferase assay was performed in AGS cells transfected with control or miR-506-3p plus WT-SNHG16 or mut-SNHG16. Data were presented as mean \pm S.D. *, $p<0.05,{ }^{\star *}, p<0.01,{ }^{\star \star *}, p<0.001$. 
A

Binding Site of hsa-miR-506-3p on PTBP1

Mutant PTBP1 3' UTR 5' GACUUGGCUUCCUUGUGGGAUAA.. Position 330-337 of PTBP1 3' UTR 5' GACUUGGCUUCCUUGUGCCUUAA.. hsa-miR-506-3p 3' AGAUGAGUCUUCCCACGGAAUG

B

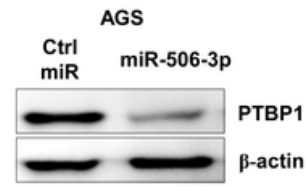

C
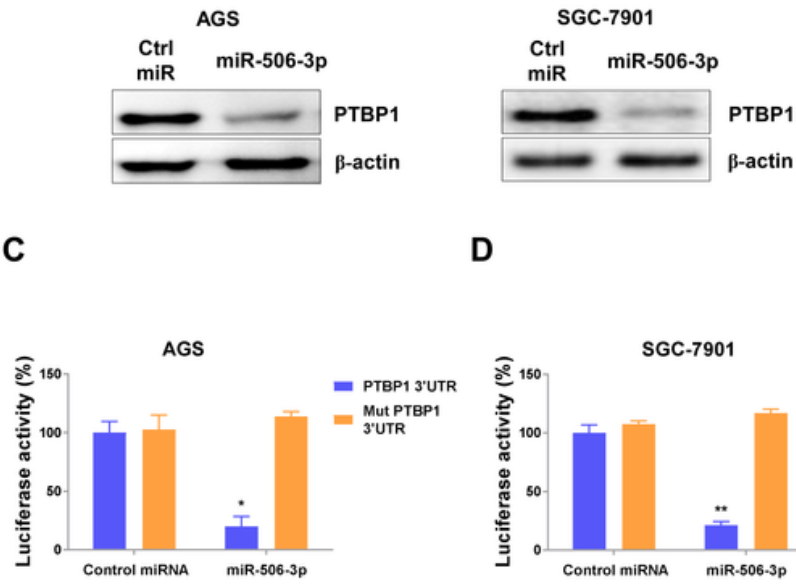

D

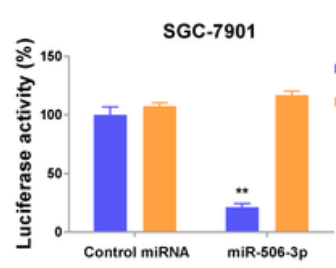

E

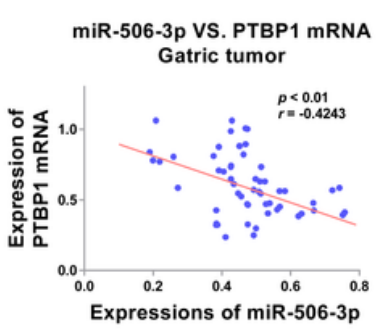

G

F

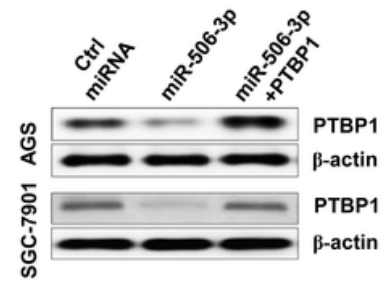

H

AGS

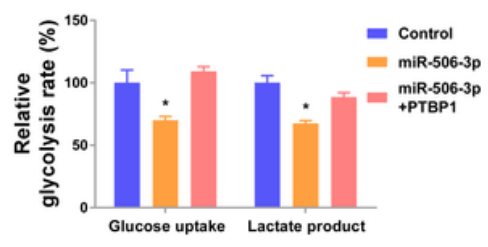

I

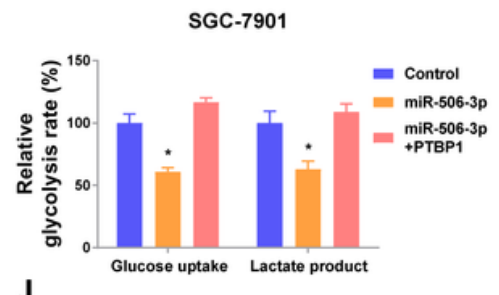

J

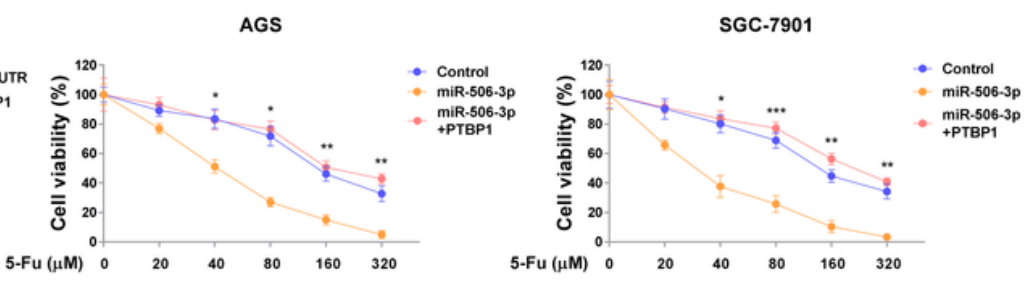

Figure 6

miR-506-3p sensitizes GC cells to 5-Fu by direct targeting PTBP1. (A) The potential binding of miR-506-3p on PTBP1 3'UTR was predicted from starBase. (B) AGS and SGC-7901 cells were transfected with control miRNA or miR-506-3p. Protein expressions of PTBP1 were determined. (C) Luciferase assay was performed in AGS and (D) SGC-7901 cells transfected with control or miR-506-3p plus WT- or mut- 3'UTR of PTBP1. (E) Pearson coefficient analysis shows a significantly negative correlation between miR-506-3p and PTBP1 in gastric tumors. (F) AGS and SGC-7901 cells were transfected with control, miR-506-3p alone or plus PTBP1. Protein expressions of PTBP1 were examined. $(G, H)$ The glucose uptake and lactate product from the above transfected cells were determined. $(\mathrm{I}, \mathrm{J})$ The above transfected cells were treated with $5-\mathrm{Fu}$ at the indicated concentrations, cell viability was determined by MTT assay. Data were presented as mean \pm S.D. ${ }^{*}, p<0.05, * \star, p<0.01, * \star \star, p<0.001$. 


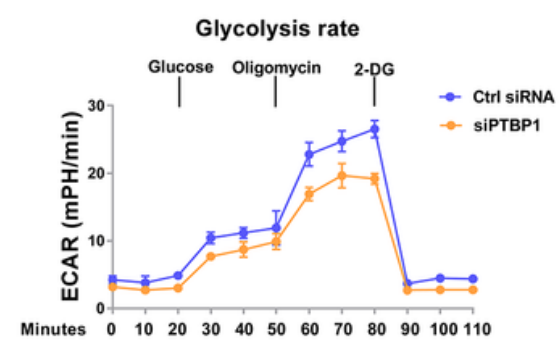

D
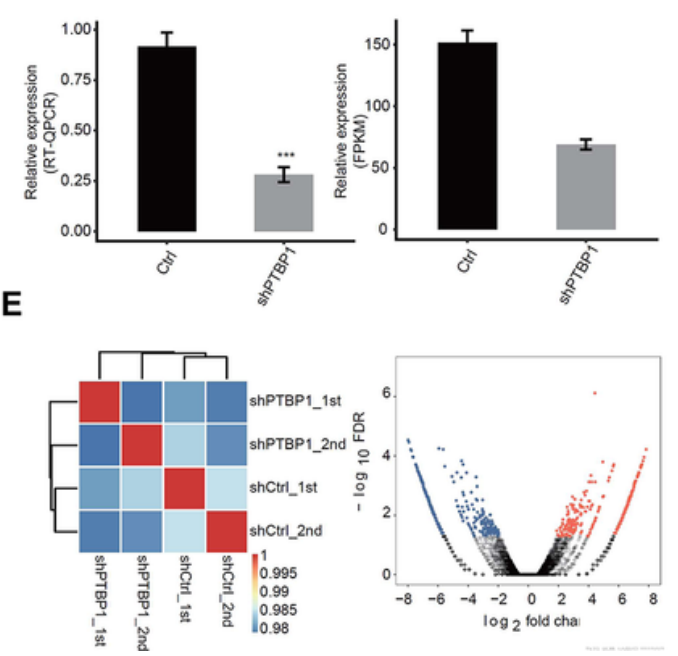



F

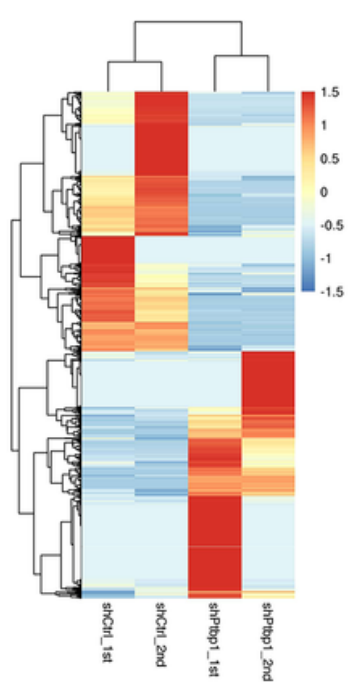

Glycolysis enzymes

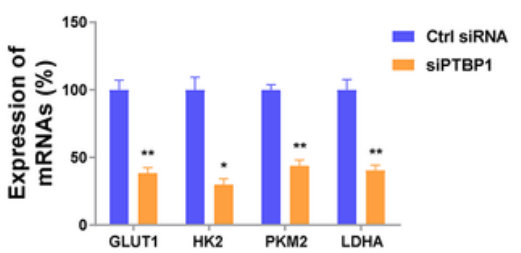

G

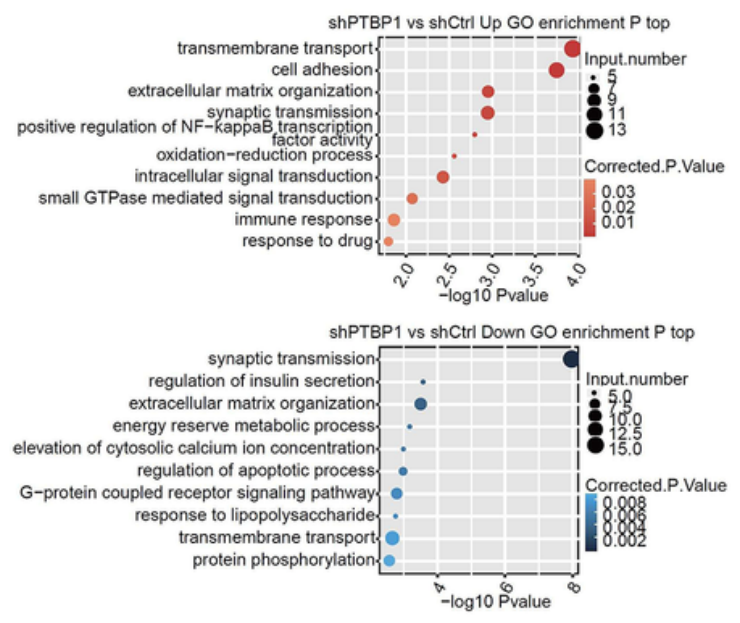

Figure 7

PTBP1 promotes glycolysis of GC cells. (A) ECAR, (B) glucose uptake and (C) mRNA expressions of glycolysis key enzymes from AGS cells without or with PTBP1 silencing. (D, E) Effects of PTBP1 knocking down by shRNA in GC cells. (F) Heatmap from transcriptome sequencing analysis shows differentially expressed mRNAs in AGS parental and 5-Fu resistant cells. Red: upregulation, Green: downregulation of gene expressions. (G) The top representatively upregulated and downregulated $\mathrm{GO}$ enrichment biological

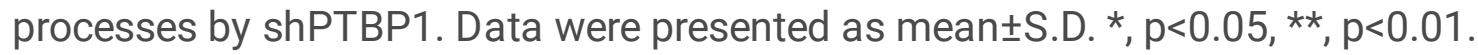

A

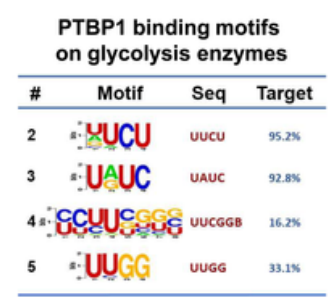

B

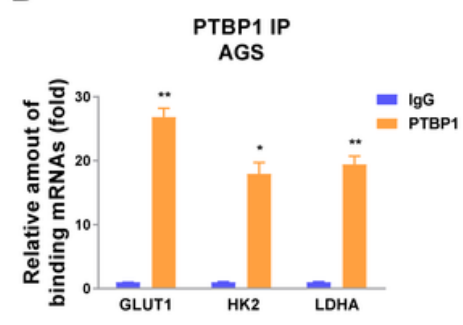

F
C



D

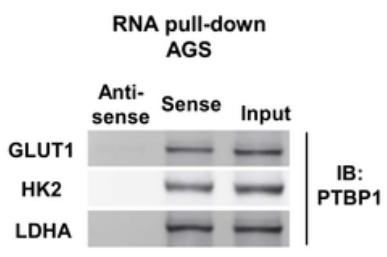

E

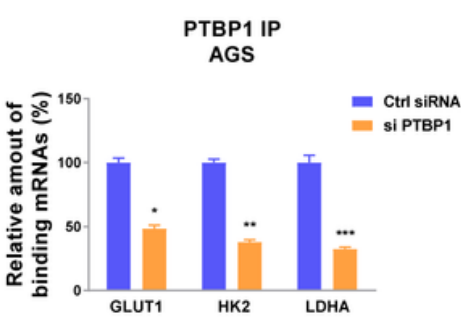

AGS



AGS

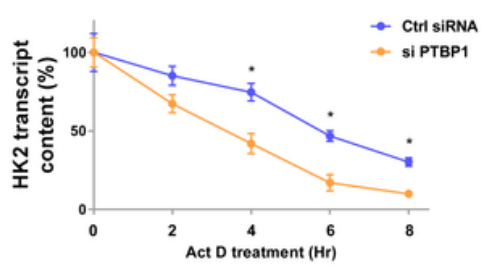

AGS

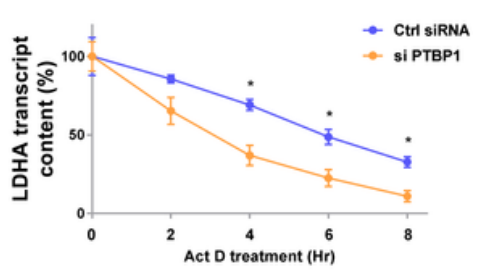




\section{Figure 8}

PTBP1 enhances the transcript stability of glycolysis enzymes by binding on 3'UTR regions. (A) Predicted PTBP1 binding motifs on glycolysis key enzymes. (B) RNA immunoprecipitation assay was performed in AGS cells using IgG control or anti-PTBP1 antibody. Glycolysis enzymes mRNA abundance in immunoprecipitated fractions was measured by qRT-PCR and (C) agarose gel electrophoresis. $\beta$-actin was an internal control. (D) RNA pull-down assay was performed in AGS cells. Biotin-labeled 3'UTR of glycolysis enzyme, GLUT1, HK2 or LDHA was incubated with cell extracts, respectively. The PTBP1 protein was assayed by western blotting. (E) AGS cells were transfected with control or PTBP1 siRNA, RNA immunoprecipitation was performed. mRNAs of glycolysis enzymes in immunoprecipitated fraction were determined by qRT-PCR. (F, G, H) AGS cells were transfected with control or PTBP1 siRNA, followed by Act $D$ treatments. Glycolysis enzymes mRNA stability assays were performed as described in Materials and Methods. Data were presented as mean \pm S.D. *, $p<0.05, * *, p<0.01, * * *, p<0.001$.

A



$E$



B

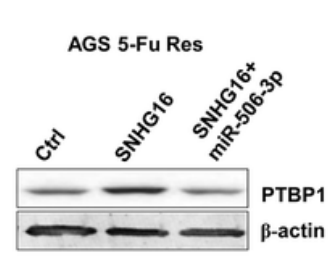

$\mathbf{F}$

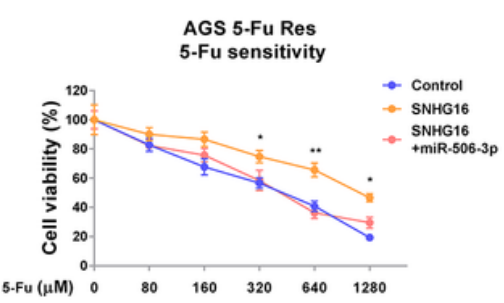

c

G


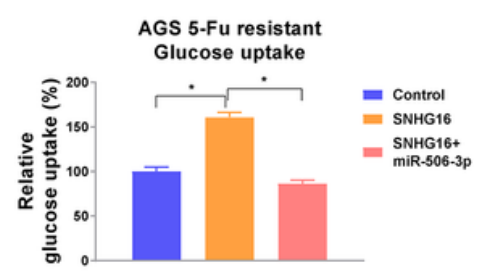

Figure 9

SNHG16 promotes 5-Fu resistance through modulating the miR-506-3p-PTBP1-glycolysis axis. (A, B) AGS 5-Fu resistant cells were transfected with control, SNHG16 alone or plus miR-506-3p. Expressions of miR506-3p and PTBP1 were examined by qRT-PCR and Western blot. (C) ECAR, (D) glucose uptake and (E) glycolysis enzymes expressions from the above transfected cells were determined. (E) The above transfected cells were treated with 5-Fu at the indicated concentrations, cell viabilities were determined by MTT and (G) Annexin V apoptosis assay. Data were presented as mean \pm S.D. *, $p<0.05, * \star, p<0.01, * \star *$, $\mathrm{p}<0.001$. 




D





E

$\mathbf{F}$

AGS 5-Fu Res

Xenograft tumor

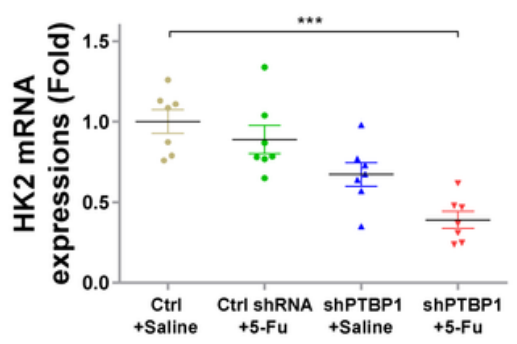

Ctrl Ctrl shRNA
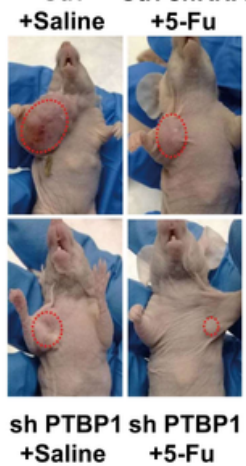

+Saline +5-Fu

AGS 5-Fu Res Xenograft tumor

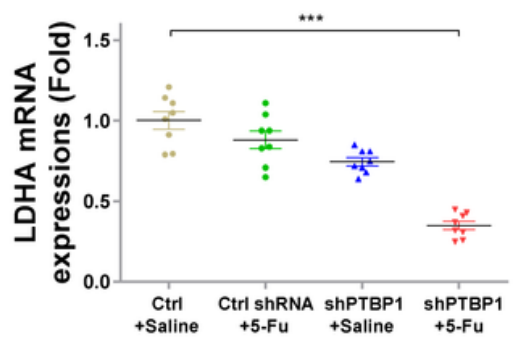

Figure 10

Inhibiting PTBP1 sensitizes gastric tumor to 5-Fu by blocking glycolysis in vivo. (A) Xenograft mice was subcutaneously injected with AGS 5-Fu resistant cells. Mice were grouped and treated with control, 5-Fu alone, shPTBP1 alone or the combination of 5-Fu and PTBP1 silencing. The survival rates of mice were recorded. (B) Xenograft tumor volumes from the above treated mice were measured and calculated. (C) Representative mice xenograft tumors from four treatment groups. (D) mRNA expressions of GLUT1, (E) HK2 and (F) LDHA from the above treated xenograft tumors were detected by qRT-PCR. Data were presented as mean \pm S.D. ***, $p<0.001$.

\section{Supplementary Files}

This is a list of supplementary files associated with this preprint. Click to download.

- Supplementalmaterials.pdf 